\title{
The role of herpesvirus entry mediator as a negative regulator of $\mathrm{T}$ cell-mediated responses
}

\author{
Yang Wang, ${ }^{1}$ Sumit K. Subudhi, ${ }^{1}$ Robert A. Anders, ${ }^{1}$ James Lo, ${ }^{1}$ \\ Yonglian Sun, ${ }^{1}$ Sarah Blink, ${ }^{1}$ Yugang Wang, ${ }^{1}$ Jing Wang, ${ }^{1}$ Xiaojuan Liu, ${ }^{1}$ \\ Karin Mink, ${ }^{2}$ Daniel Degrandi, ${ }^{3}$ Klaus Pfeffer, ${ }^{3}$ and Yang-Xin Fu ${ }^{1}$
}

\begin{abstract}
1Department of Pathology, University of Chicago, Chicago, Illinois, USA. ${ }^{2}$ Institute of Medical Microbiology, Immunology, and Hygiene, Technical University of Munich, Munich, Germany. ${ }^{3}$ Institute of Medical Microbiology, Heinrich-Heine-Universität Düsseldorf, Düsseldorf, Germany.
\end{abstract}

\begin{abstract}
Herpesvirus entry mediator (HVEM), a TNF receptor superfamily member, has been previously described as a $\mathrm{T}$ cell costimulatory receptor. Surprisingly, $\mathrm{HVEM}^{-/-} \mathrm{T}$ cells showed enhanced responses to in vitro concanavalin $\mathrm{A}$ (ConA) stimulation when compared with WT T cells. Consistent with these findings, $H V E M^{-/-}$mice exhibited increased morbidity and mortality as compared with WT mice in a model of ConA-mediated T cell-dependent autoimmune hepatitis. $\mathrm{HVEM}^{-/-}$mice produced higher levels of multiple cytokines, which were dependent on the presence of $\mathrm{CD}^{+} \mathrm{T}$ cells. Furthermore, $\mathrm{HVEM}^{-/-}$mice were more susceptible to MOG peptide-induced experimental autoimmune encephalopathy, and they showed increased $T$ cell proliferation and cytokine production in response to antigen-specific challenge. Taken together, our data revealed an unexpected regulatory role of HVEM in $T$ cell-mediated immune responses and autoimmune diseases.
\end{abstract}

\section{Introduction}

The engagement of an antigen-specific TCR with the MHC/ peptide complex displayed on APCs provides the first essential signal for $T$ cell activation. These TCR-mediated signals modulated by cosignaling molecules expressed on the cell surface result in augmentation (costimulation) of T cell responses (1-3). Several B7 family members have been identified as possessing costimulatory activity, which may play important roles in various $\mathrm{T}$ cell-mediated responses, such as autoimmunity and organ transplantation (4). The temporal and spatial expressions of the cosignals regulate priming, growth, differentiation, and functional maturation of $\mathrm{T}$ cell-mediated immune responses (1-3).

In addition to the B7 family, some TNF superfamily members can also play a costimulatory role in $\mathrm{T}$ cell activation $(1,2)$. LIGHT (TNFSF14), a newly identified costimulatory ligand, is predominantly expressed on lymphoid tissues, especially on the surface of activated DCs and T cells (5-7). LIGHT is a potent $\mathrm{T}$ cell costimulatory molecule that appears to interact with the herpesvirus entry mediator (HVEM) expressed on T cells $(5,6,8)$. Unlike most other costimulatory molecules delivering signals from APCs, the upregulation of LIGHT on T cells enhanced $\mathrm{T}$ cell-mediated immunity and promoted autoimmune diseases and inflammation (9-11). Local expression of LIGHT on tumor tissues induced a robust expansion and activation of $\mathrm{T}$ cells that led to the rejection of local and distal tumors (12). Conversely, $\mathrm{LIGHT}^{-/-}$mice exhibited impaired T cell responses (13-15). Transient transfection of HVEM into human 293 cells caused marked activation of NF- $\mathrm{\kappa B}$, a transcriptional regulator

Nonstandard abbreviations used: ALT, alanine aminotransferase; AST, aspartate aminotransferase; BTLA, B and T lymphocyte attenuator; ConA, concanavalin A; DLN, draining lymph node; EAE, experimental autoimmune encephalopathy; HVEM, herpesvirus entry mediator.

Conflict of interest: The authors have declared that no conflict of interest exists.

Citation for this article: J. Clin. Invest. 115:711-717 (2005)

doi:10.1172/JCI200522982. of multiple immunomodulatory and inflammatory genes (16). Recent studies have suggested that LIGHT-HVEM signaling is required for stronger $T$ cell-mediated immunity $(9-11,17)$. To directly study the essential role of HVEM in T cell activation, we generated $H V E M^{-/-}$mice. Unexpectedly, $H V E M^{-/-}$mice exhibited enhanced responses to various $\mathrm{T}$ cell stimuli, both in vivo and in vitro, and were more susceptible to developing autoimmune diseases, thereby revealing another potential function of HVEM as a possible inhibitor of $\mathrm{T}$ cell responses.

\section{Results}

The generation and analysis of HVEM KO mice. To directly define the essential function of HVEM in vivo, we generated $\mathrm{HVEM}^{-/-}$mice by genetic targeting as illustrated in Figure 1A. Southern blot analysis showed disruption of the gene encoding HVEM in both ES cells and mice after germ-line transmission of the HVEM mutation (Figure 1B). Northern blot analysis of RNA from WT and $\mathrm{HVEM}^{-1-}$ splenic tissues (Figure $1 \mathrm{C}$ ) and flow cytometric analysis (Figure 1D) of peripheral blood lymphocytes from WT and $\mathrm{HVEM}^{-/-}$mice confirmed that the target allele encoding HVEM had a null mutation. Almost all WT peripheral blood lymphocytes stained positively for HVEM. Double staining of lymph node cells from WT mice with anti-CD3 and anti-HVEM mAbs revealed that $\mathrm{CD}^{+}$cells expressed higher levels of HVEM than non-T cell populations. The expression of HVEM was completely absent in $H V E M^{-/-}$mice in both cell types (Figure 1D). The litters from $H_{V E M}{ }^{-/-}$mice were born at the expected frequency and size with normal maturation and fertility. T, B, NK, and DC populations in the thymus, lymph nodes, and spleen were normal (Figure 1E and data not shown). In addition, IgM and IgG antibody production in response to keyhole limpet hemocyanin and sheep rbc immunization appeared to be normal in $H V E M^{-/-}$mice (data not shown). Similar to $\mathrm{LIGHT}^{-/-}$mice, $H V E M^{-/-}$mice exhibited no defects in the organogenesis of lymphoid tissues and lymphocyte development. 
A Genomic locus of murine HVEM

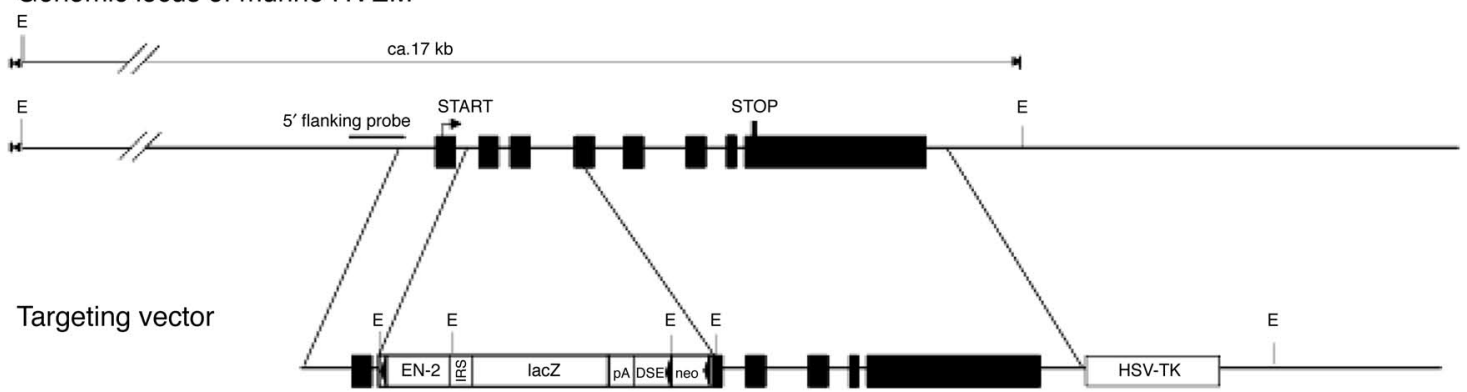

Targeted allele

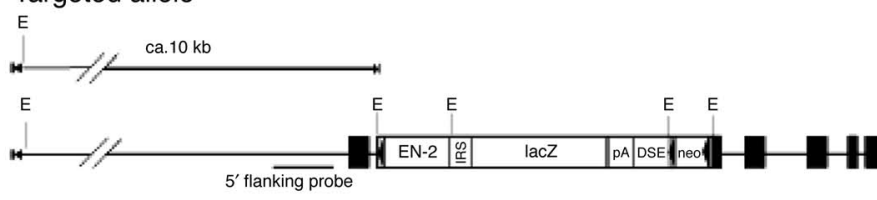

B

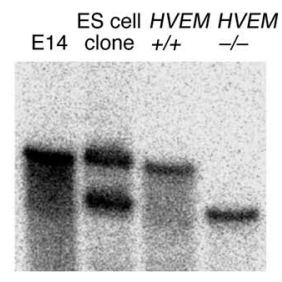

C

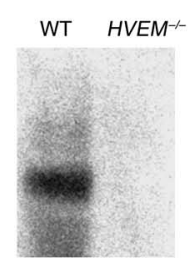

D

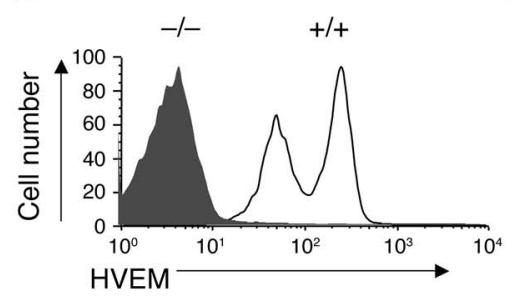

$\mathbf{E}$

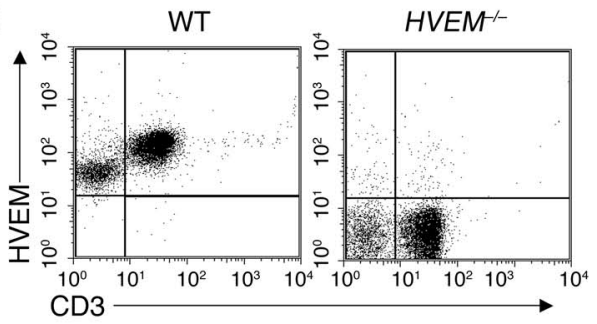

Figure 1

Targeted disruption of the HVEM locus. (A) Schematic map of the HVEM WT locus, the targeting vector, and the inactivated $H V E M$ allele. Black boxes indicate the exons of the HVEM gene. The expected fragment size after hybridization with the $5^{\prime}$ flanking probe (EcoRI digest of genomic DNA) is approximately $17 \mathrm{~kb}$ for the WT allele and $10 \mathrm{~kb}$ for the inactivated allele. EN-2, engrailed-2 splice acceptor site; lacZ, $\beta$-gal expression cassette; pA, polyadenylation signal; DSE, downstream element; neo, neomycin resistance gene cassette; HSV-TK, herpesvirus 1 thymidine kinase expression cassette. (B) Southern blot analysis of mice after germ-line transmission of the HVEM mutation. Hybridization of EcoRI-digested tail DNA of representative mice is shown (E14, control DNA from E14.1 ES cells). (C) Northern blot analysis of HVEM mRNA. Hybridization of spleen RNA is depicted using a murine HVEM cDNA encompassing the complete coding sequence for the extracellular domain. (D) Blood was collected from C57BL/6 (WT) and $H_{V E M}-1-$ mice, and white blood cells were stained with biotin-labeled rat anti-HVEM mAb and streptavidin-CyChrome. (E) Lymph node cells from WT and $H_{V E M}{ }^{--}$mice were stained with PE-anti-CD3, biotin-labeled rat anti-HVEM antibody, and streptavidin-CyChrome.

Enhanced activation of $H V E M^{-1-}$ T cells in vitro and in vivo. Though several studies have suggested that HVEM may have $\mathrm{T}$ cell costimulatory function (5-7), the role of HVEM in T cell activation has never been directly demonstrated. To test whether HVEM is required for CD3-mediated activation, splenocytes isolated from WT or $H V E M^{-/}$mice were stimulated with plate-bound anti-CD3 mAb. Unexpectedly, the data showed that HVEM-deficient splenocytes responded to anti-CD3 mAb stimulation better than WT splenocytes did (data not shown). To determine whether HVEM deficiency has a direct impact on T cells, purified T cells (more than 98\% pure) from both WT and $\mathrm{HVEM}^{-/-}$mice were stimulated by immobilized anti-CD3 mAb. HVEM-deficient T cells had a stronger proliferative response than WT T cells (Figure 2A), confirming that the hyperresponsiveness observed in HVEM-deficient splenocytes was intrinsic to the $T$ cell population and can occur in the absence of APCs. These results suggest that HVEM might play a direct negative role in modulating $T$ cell activity.

To further investigate the response of $H V E M^{-1-} \mathrm{T}$ cells to other T cell stimuli, splenocytes from WT and $H V E M^{-/-}$mice were cultured with different doses of concanavalin A (ConA). Consistently, $H V E M^{-1-} \mathrm{T}$ cells showed increased proliferation compared with WT T cells in response to ConA (Figure 2B). Furthermore, these T cells produced higher levels of cytokines in the culture (Figure 2C).
To determine whether HVEM-deficient T cells were more active, purified T cells from WT and $H V E M^{-/-}$mice were stimulated with ConA in the presence of either WT or HVEM-deficient APCs. HVEM-deficient $T$ cells again showed higher proliferation in response to ConA stimulation compared with WT T cells (Figure 2D). Interestingly, APCs from $\mathrm{HVEM}^{-/-}$mice were more active in stimulating $\mathrm{T}$ cells than those from WT mice. These results support that HVEM might be a negative regulator of $\mathrm{T}$ cell activation, which is in stark contrast to its costimulation activity in $\mathrm{T}$ cell activation, presented in previous studies.

We further investigated whether LIGHT, a well-defined ligand of HVEM, is involved in HVEM-mediated negative regulation of $\mathrm{T}$ cell activation. Equal numbers of WT, $H V E M^{-/-}$, and $\mathrm{LIGHT}^{-/-}$ splenocytes were cultured in the presence of ConA, and T cell proliferation and cytokine levels in those culture supernatants were measured. Though the approximate $T$ cell numbers in the spleens of these mice were very similar, splenocytes from $\mathrm{HVEM}^{-/-}$mice but not from $L I G H T^{-1-}$ mice exhibited increased $\mathrm{T}$ cell proliferation and increased cytokine production as compared with WT splenocytes in response to ConA (Figure 2E). The data strongly suggested that LIGHT may not be the ligand responsible for HVEM-mediated negative regulation of $\mathrm{T}$ cell activation and that LIGHT might not be the sole molecule interacting with HVEM. 
A

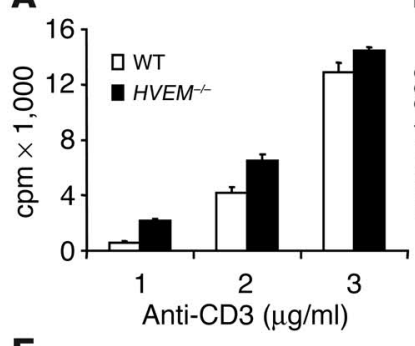

$\mathbf{E}$

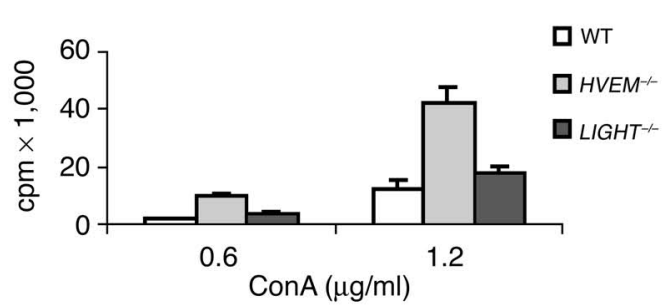

B

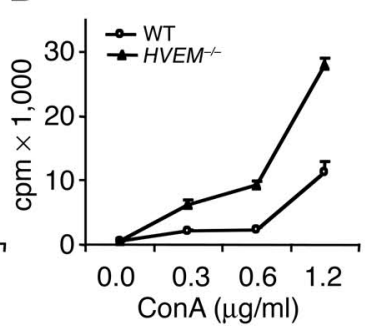

C
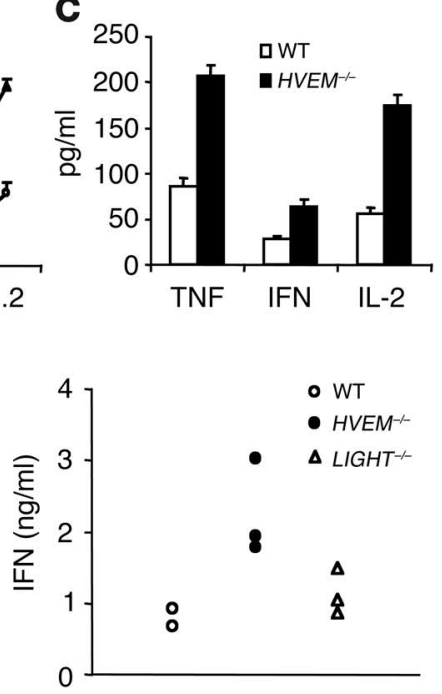

D

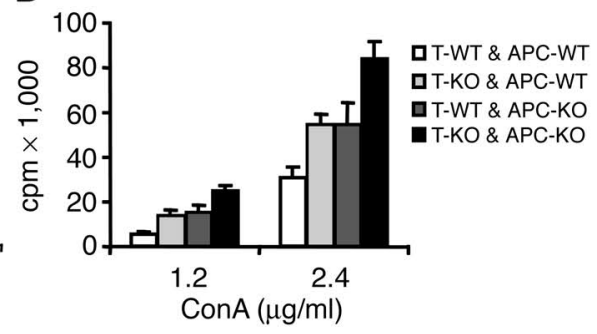

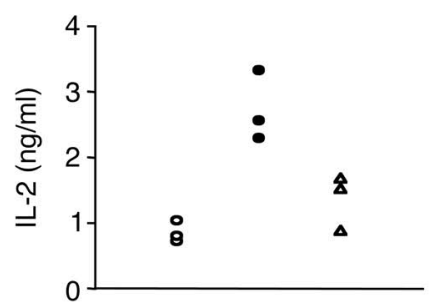

Figure 2

Overreactive HVEM-deficient T cells in vitro. (A) HVEM-l- T cells are more sensitive to stimulation by suboptimal anti-CD3 mAb as shown by ${ }^{3} \mathrm{H}$-thymidine incorporation assay. Data represent ${ }^{3} \mathrm{H}$-thymidine incorporation in $\mathrm{cpm} \pm \mathrm{SD}$. One of 4 independent experiments is shown. (B) Splenocytes $\left(2 \times 10^{5}\right.$ per well) from WT and $H V E M^{-1-}$ mice were cultured in 96 -well plates with $0,0.3,0.6$, and $1.2 \mu \mathrm{g} / \mathrm{ml}$ ConA in vitro. $H_{V E M}{ }^{-1-}$ mice showed significantly increased response to ConA at all concentrations $(P<0.01)$. Data represent 1 of 6 independent experiments. (C) Cytokine levels in culture supernatants from B were determined by cytokine bead assay. The cells from $H V E M^{-1-}$ mice also produced significantly higher levels of cytokines $(P<0.001)$. Representative data are shown from 1 of 3 independent experiments. (D) $H V E M$ deficiency in both T cells and APCs contributes to the higher T cell responses to ConA. T cells from $H V E M^{-1-}$ mice (T-KO) showed higher proliferation than T cells from WT mice in response to ConA when APCs from WT mice were provided $(P<0.01)$, and APCs from $H V E M{ }^{-/-}$mice $($APC-KO) also provided better stimulation than APCs from WT mice $(P<0.01)$ when T cells from WT mice were used as responders. Representative data are shown from 3 independent experiments. (E) Increased $H V E M^{-1-}$ T cell response but not $L I G H T^{-1-}$ T cell response to ConA in vitro. Splenocytes from WT HVEM ${ }^{-/-}$and $\mathrm{LIGHT}^{-/-}$mice were collected and cultured with ConA. The proliferation was determined by ${ }^{3} \mathrm{H}$-thymidine incorporation assay $(P<0.01)$, and cytokine levels were determined by cytokine bead assay $(P<0.05)$.

Administration of ConA into WT mice leads to systemic T cell activation and $\mathrm{T}$ cell-mediated hepatitis. To evaluate the role of HVEM in $\mathrm{T}$ cell activation in vivo, WT and $\mathrm{HVEM}^{-/-}$mice were injected i.v. with a sublethal dose of ConA $(16 \mathrm{mg} / \mathrm{kg})$. Surprisingly, 6 of 7 $\mathrm{HVEM}^{-/-}$mice died within 8 hours, while all of the WT mice (7 of 7) survived for at least 24 hours (Figure 3). In a total of 6 independent experiments, 15 of $28 \mathrm{HVEM}^{-1-}$ mice died within 8 hours, while 0 of 28 WT mice died. To compare the role of LIGHT and HVEM in vivo, we challenged WT, HVEM ${ }^{-/-}$, and LIGHT $^{-/-}$mice with sublethal doses of ConA $(16 \mathrm{mg} / \mathrm{kg})$. In this experiment, $H_{V E M^{--}}$mice (5 of 5) were very sick within 3 hours after the administration of ConA and began to die within 4 hours, while none of the WT mice (0 of 5) or $\mathrm{LIGHT}^{-1-}$ mice ( 0 of 5 ) were sick (data not shown). To investigate whether $H V E M^{-/-}$mice developed more severe hepatitis, sera collected from those ConA-treated WT and $\mathrm{HVEM}^{-/-}$mice at different time points were measured for alanine aminotransferase (ALT) levels. We detected no significant differences in elevated ALT levels in either group of mice (Figure 4A). H\&E and TUNEL staining of liver tissues from both groups of mice showed low levels of infiltrating leukocytes and equally low numbers of cells undergoing apoptosis or necrosis in the livers (data not shown). These data suggested that the increased morbidity and mortality of $\mathrm{HVEM}^{-/-}$mice were unlikely to have been caused solely by liver damage.

To assess overall tissue damage, serum aspartate aminotransferase (AST) levels in WT and HVEM ${ }^{-/-}$mice after treatment with ConA were measured and showed an equal increase by 6 hours in both groups of mice (Figure 4B). To test whether ConA caused T cell hyperreactivity in the absence of HVEM, splenic sections from ConA-treated $\mathrm{HVEM}^{-1-}$ and WT mice were evaluated for potential tissue damage by H\&E staining. HVEM-/- mice showed more severe cellular loss and increased apoptosis in the white pulp areas, the $\mathrm{T}$ cell zone in particular, of the spleen at 8 hours following ConA injection (Figure 4C). These results suggested that the increased morbidity in $\mathrm{HVEM}^{-/-}$mice may not be due primarily to liver damage but rather may be due to overreactive splenocytes.

Elevated serum cytokines in $H V E M^{-/}$mice after ConA administration. To test whether the splenocytes from $\mathrm{HVEM}^{-/-}$mice were hyperreactive after ConA administration, sera from both groups of mice were collected at 2 and 6 hours after ConA administration and were measured for the levels of various cytokines, such as TNF- $\alpha$, IFN- $\gamma$, IL-2, and IL-6, by ELISA and/or cytokine bead assay. HVEM ${ }^{-/-}$mice showed significantly higher and prolonged levels of cytokines in the sera between 2 and 6 hours following ConA treatment (Figure 5). Such high and prolonged levels of cytokines might contribute to increased morbidity and mortality in $\operatorname{HVEM}^{-/-}$mice $(18,19)$. Therefore, our in vitro and in vivo data together indicated that $\mathrm{HVEM}^{-/-} \mathrm{T}$ cells might be more active in generating cytokines in response to ConA stimulation.

$C D 4^{+}$cells were responsible for hyperreactivity. ConA-mediated cytokine production depends on the interactions between $\mathrm{CD}^{+} \mathrm{T}$ cells and NKT cells (20). Early cytokine production in $H_{V E M^{-1-} \mathrm{T}}$ cells might be mostly attributed to $\mathrm{CD} 4^{+}$cells, which include both the NK1.1 ${ }^{+}$and the NK1.1- $\mathrm{T}$ cell populations. To test this possibility, 1 group of $\mathrm{HVEM}^{-/-}$mice was treated with $100 \mu \mathrm{g}$ anti-CD4 


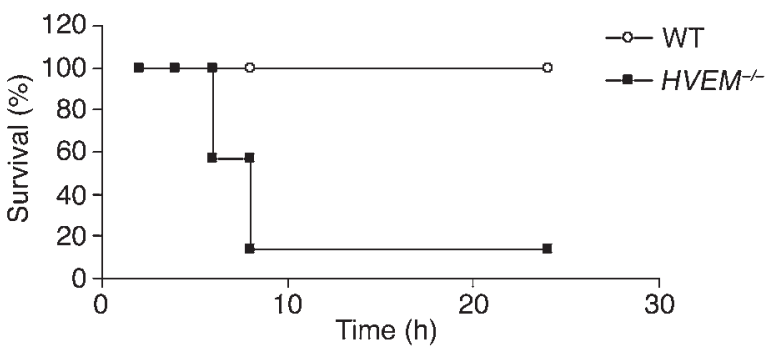

Figure 3

$H V E M^{-1-}$ mice are more sensitive to ConA challenge in vivo. Sublethal ConA (16 mg/ml) was injected i.v. into age- and sex-matched WT and $H V E M^{-/-}$mice ( $n=7,3-5$ months old). The mortality and morbidity of 2 groups of mice were recorded hourly for 24 hours after ConA injection. One of 4 experiments is shown.

$\mathrm{mAb} 1$ day before ConA administration to deplete $\mathrm{CD}^{+} \mathrm{T}$ cells. Following ConA treatment, $H_{V E M^{-/-}}$mice were sick and dying by 6-8 hours, whereas CD4-depleted $H V E M^{-/-}$mice showed no signs of sickness for more than a 48-hour period. Serum cytokines from WT, $\mathrm{HVEM}^{-/-}$, and CD4 ${ }^{+} \mathrm{T}$ cell-depleted $\mathrm{HVEM}^{-/-}$mice were measured. Interestingly, the phenomenon of increased cytokines in $\mathrm{HVEM}^{-/-}$mice after ConA stimulation was diminished in CD4depleted $H V E M^{-/-}$mice, which suggested that $\mathrm{CD}^{+} \mathrm{T}$ cells were the essential source for rapidly elevated cytokines in $\mathrm{HVEM}^{-/-}$mice (Figure 6). Taken together, these results suggested that $\mathrm{CD}^{+} \mathrm{T}$ cells were essential for the elevated cytokine production in $\mathrm{HVEM}^{-/-}$ mice in response to ConA treatment.

Increased susceptibility to the development of experimental autoimmune encephalopathy in $\mathrm{HVEM}^{-/-}$mice. To assess whether $\mathrm{HVEM}^{-/-}$mice were more susceptible to developing $T$ cell-mediated autoimmune diseases such as experimental autoimmune encephalopathy (EAE), WT and $H V E M^{-/-}$mice were immunized with MOG peptide emulsified in CFA. Interestingly, the results showed that $\mathrm{HVEM}^{-1-}$ mice developed EAE earlier and for a prolonged period as compared with WT mice (Figure 7A). Recovery from the disease was not observed in most $\mathrm{HVEM}^{-/-}$mice up to 32 days after disease onset, whereas WT mice recovered much sooner. To address whether the increased severity of EAE in $\mathrm{HVEM}^{-/-}$mice was associated with increased T cell responses to antigen, draining lymph node (DLN) cells were isolated from the primed mice 20 days after s.c. immunization with MOG 35-55 peptide (without the toxin) and were restimulated with MOG 35-55 peptide in vitro. DLN cells from $H V E M^{-/-}$mice proliferated more than those from WT DLNs (Figure 7B) and produced higher levels of TNF- $\alpha$ and IFN- $\gamma$ (Figure 7C). The frequency of antigen-specific T cells among the DLN cells of primed $H V E M^{-1}$ mice was much higher than that in WT mice as determined by ELISPOT (Figure 7D). These results indicated that $\mathrm{HVEM}^{-/-}$mice were more susceptible to developing autoimmune EAE, which further supports that HVEM negatively regulated T cells in vivo.

\section{Discussion}

The data from many previous studies suggested that the costimulatory activity of LIGHT was mediated by its engagement with HVEM, a receptor expressed on T cells: (a) LIGHT on both $\mathrm{DC}$ and $\mathrm{T}$ cells has been shown to augment $\mathrm{T}$ cell proliferation and cytokine production (5-7). (b) Recombinant LIGHT can directly costimulate $T$ cell responses (21). (c) Mice expressing the LIGHT transgene on the $T$ cell lineage exhibited severe $T$ cell-mediated inflammation and autoimmune diseases $(9,11)$. (d) Blockade of LIGHT by HVEM-Ig reduced T cell proliferation and the incidences of T cell-mediated diseases (11). (e) LIGHT ${ }^{-1-}$ mice showed defective $T$ cell responses in vivo and in vitro (13-15). These data supported the theory that HVEM is a receptor for LIGHTmediated $T$ cell activation. In this study, however, we have demonstrated that, in the absence of HVEM-mediated signaling, $\mathrm{T}$ cells exhibited increased responses to various in vitro and in vivo stimuli: (a) $H V E M^{-1-}$ T cells were hyperreactive to anti-CD3 mAb and ConA stimulation in vitro; (b) administration of ConA into WT and $H V E M^{-/-}$mice rapidly resulted in higher production of proinflammatory cytokines and higher morbidity and mortality in $\mathrm{HVEM}^{-/-}$mice than in WT mice, within 4-8 hours; and (c) $\mathrm{HVEM}^{-/-}$ mice were more susceptible to the induction of autoimmunity. Our preliminary data showed that $H V E M^{-/-}$mice also cleared listerial infection faster than WT mice (data not shown). Together, our data suggest a new model in which signaling via HVEM on T cells might negatively regulate $T$ cell-dependent immune responses.

It is increasingly becoming recognized that molecules initially described as costimulatory ligands can also have coinhibitory activity when different receptors are engaged. B7-1 (CD80) or B7-2 (CD86) ligation represents a well-defined example of this by using
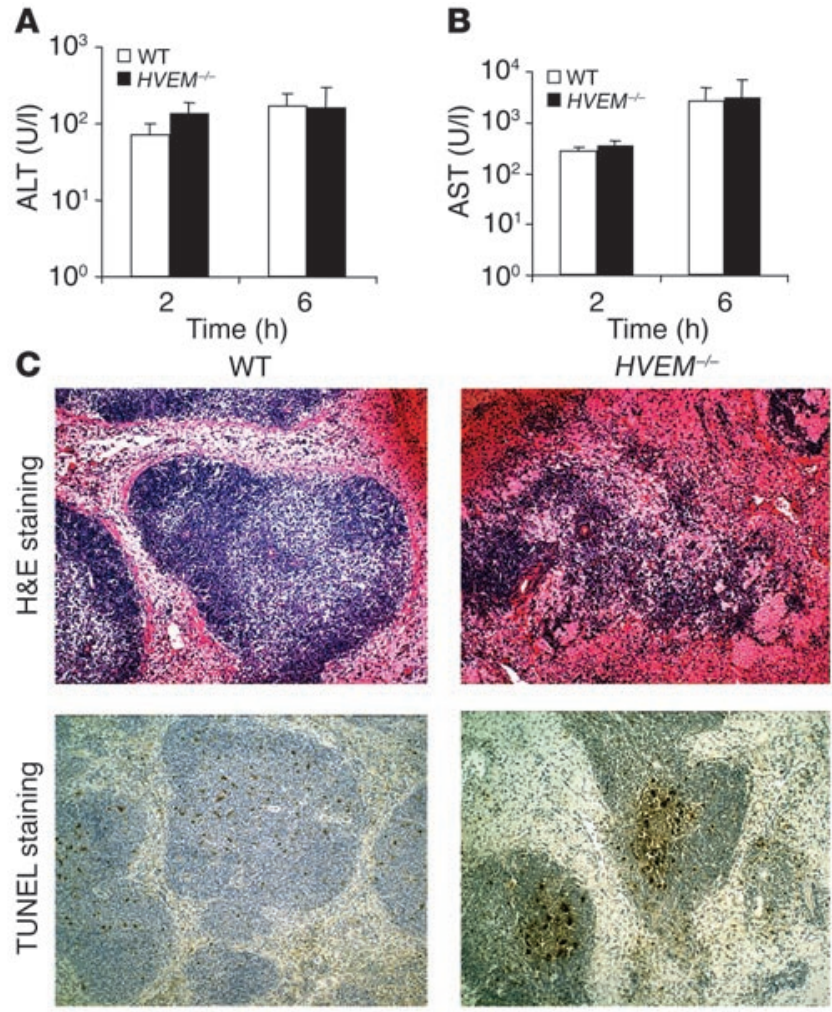

\section{Figure 4}

Limited liver damage and severe spleen damage occur after ConA administration. (A and B) Sera from WT and HVEM-1- mice were collected at 2, 6, and 8 hours after ConA injection. Serum ALT and AST levels were determined. $H V E M^{-/-}$mice showed similar levels of ALT (A) and AST (B) to those of WT mice. (C) Histological examination of WT and $H V E M^{-/-}$mice after ConA injection. The spleen sections were stained with H\&E and TUNEL. The spleen sections of $H V E M^{-1-}$ mice showed massive loss of splenocytes and increased clusters of apoptosis in the T cell zone. 

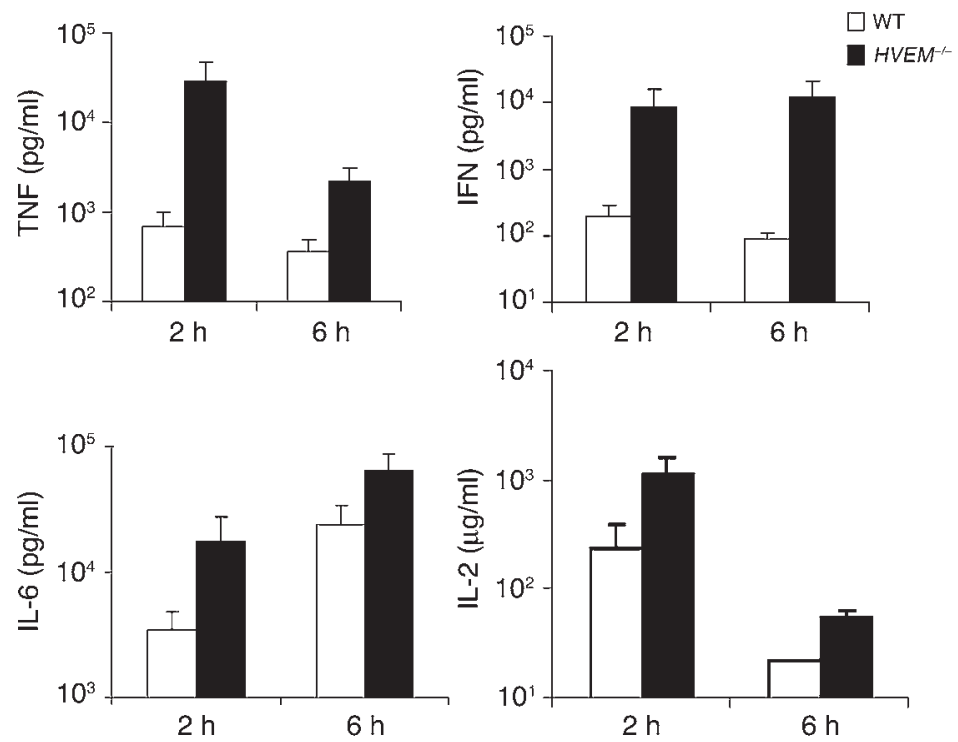

Figure 5

Administration of ConA increases serum cytokines in $H V E M^{-/-}$mice. Sera from WT and KO mice were collected at 2 and 6 hours after ConA administration. TNF, IFN, IL-6, and IL-2 in sera were determined by sandwich ELISA, and statistically significant differences were shown in all groups at both 2 and 6 hours. with HVEM-deficient T cells. In support of this idea, we have found that HVEM-Ig binds to an undefined ligand on B cells from LIGHT-deficient mice. In addition, plate-bound HVEM-Ig inhibited anti-CD3-mediated $\mathrm{T}$ cell proliferation in a LIGHT-independent fashion (data not shown). These data suggest that HVEM may deliver negative signaling to limit CD3-mediated $\mathrm{T}$ cell response. After we revised our manuscript, a recent study by Murphy's group showed that HVEM binds to B and T lymphocyte attenuator (BTLA) (34). The study further showed that HVEM is a unique BTLA ligand. BTLA bound the most membrane-distal cysteine-rich domain of HVEM, distinct from regions where the ligands LIGHT and lymphotoxin- $\alpha$ bound HVEM. Furthermore, HVEM induced BTLA tyrosine phosphorylation and association of the tyrosine phosphatase SHP-2 and repressed antigen-driven $T$ cell proliferation. Together, these findings indicated that HVEM might provide negative feedback mechanisms for regulating $T$ cell responses, in contrast to its positive role in $\mathrm{T}$ cell costimulation described in other studies. Therefore, HVEM may play dual roles in T cell activation and homeostasis, depending on the ligand and receptor engagement during immune responses. It will be interesting to determine how HVEM can receive and deliver the different signaling in the future.
2 different receptors, CD28 and T lymphocyte associate antigen-4 (CTLA-4). CD28 engagement by B7-1 and B7-2 augmented TCRmediated signals for prolonged $\mathrm{T}$ cell survival, clonal expansion, and differentiation $(3,22)$, whereas the interactions of B7-1 and B7-2 with CTLA-4 resulted in the inhibition of $\mathrm{T}$ cell responses $(4,23)$. In fact, other B7 family members have been found to be important $\mathrm{T}$ cell costimulators and coinhibitors. In most cases, many of these B7 family members could deliver either a positive or a negative signal on $\mathrm{T}$ cells by binding to different receptors (2). Though B7-H1 (PD-L1) expressed in multiple nonlymphoid tissues was initially found to be costimulatory $(24,25)$, several subsequent studies have demonstrated that the interaction of B7-H1 with PD-1 led to the inhibition of T cell responses $(26,27)$. Recent studies have also provided evidence for the costimulatory effects of B7-H1, which is likely mediated by binding to a receptor other than PD-1 $(2,28)$. B7-H3 has also been shown to have both $\mathrm{T}$ costimulatory and coinhibitory functions, although its receptors have not yet been defined $(29,30)$. The mechanisms underlying the discrepancies are unclear. Some TNF family members showed similar dual functions: for example, recent studies showed that agonistic anti-CD137 (4-1BB, another TNF receptor superfamily member) might promote antitumor activity while ameliorating autoimmunity (31-33).

While our study does not exclude that HVEM is an important receptor for LIGHT-mediated costimulation, LIGHT may not be the only ligand for HVEM, since LIGHT-deficient T cells did not display hyperreactivity to ConA stimulation in vitro in comparison

\section{Methods}

Mice. C57BL/6 mice were purchased from The Jackson Laboratory. The mice were maintained under specific pathogen-free conditions. Animal care and use were in accordance with institutional and NIH protocols and guidelines, and all studies were approved by the Animal Care and Use Committee of the University of Chicago. LIGHT ${ }^{-1-}$ mice were kindly provided by Lieping Chen (Johns Hopkins University, Baltimore, Maryland, USA) (13-15).

Generation of HVEM-deficient mice. A genomic bacterial artificial chromosome (BAC) clone containing the HVEM locus was obtained from Genome Systems Inc. by screening of a BAC library with HVEM-specific primers, derived from the HVEM mRNA sequence (muHVEM2: 5'-ATGGCCTGAGCAAGTGTCTGC-3'; and Int3Rev: 5'-ACACACCCTGAGAACCTGCCCAC-3'). An approximately 8.8 -kb genomic subclone (with HindIII restriction sites) coding $H V E M$ was subcloned and completely sequenced. The targeting vector was constructed using homologous fragments of the HVEM locus (see Figure 1). Exons 2 and 3 and the $5^{\prime}$ region of exon 4 were replaced by an intron gene

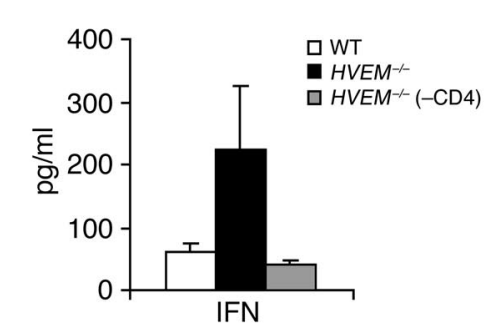

Figure 6

$\mathrm{CD}^{+} \mathrm{T}$ cells are required for higher cytokine production in $\mathrm{HVEM}^{---}$mice. To delete CD4 ${ }^{+} \mathrm{T}$ cells, $100 \mu \mathrm{g} /$ mouse anti-CD4 mAb was injected i.p. into $\mathrm{HVEM}^{-1-}$ mice $(n=5)$. Sera were collected from 5 mice per group 4-5 hours later, and IFN, TNF, and IL-2 were determined by cytokine bead assay. Depletion of CD4+ cells significantly reduced the levels of cytokines $(P<0.05$ for IFN and TNF; $P<0.01$ for IL-2). 


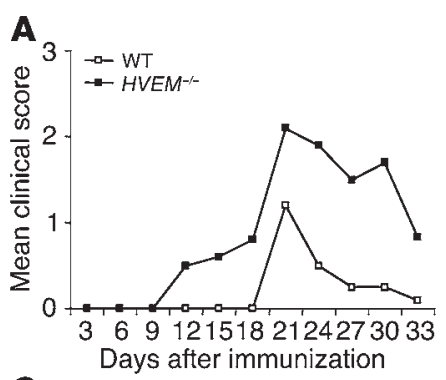

c
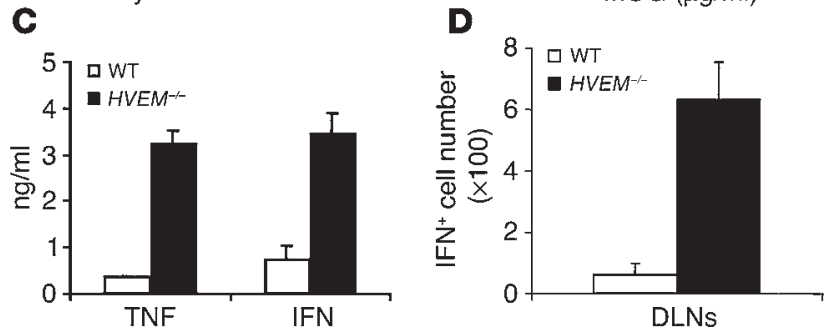

Figure 7

Increased susceptibility to developing EAE in $\mathrm{HVEM}^{-/-}$mice. Development of EAE was induced in WT mice and $\mathrm{HVEM}^{-/-}$mice as described in Methods. (A) Mice were observed daily and scored. The mean clinical score plotted against time is shown. Five mice per group were tested. (B-D) Stronger MOG-specific T cell responses are detected in $\mathrm{HVEM}^{-1-}$ mice. The mice were immunized s.c. with $100 \mu \mathrm{g}$ of MOG 35-55 emulsified in CFA (without toxin). Ten days later, DLN cells from the mice were isolated and cultured with various concentrations of MOG 35-55 peptide in complete RPMI-1640 medium for 5 days for proliferation analysis (B). Stronger responses were detected in the cells from the $H_{V E M}{ }^{--}$group at concentrations of 1,5 , and $25 \mu \mathrm{g}(P<0.05)$. The number of IFN-producing cells was determined by ELISPOT (C). Cells producing higher levels of IFN were detected in $\mathrm{HVEM}^{-1-}$ mice $(P<0.001)$. DLN cells were stimulated with antigens overnight for ELISPOT to determine the frequency of antigen-specific $T$ cells (D). DLN cells from $\mathrm{HVEM}^{-/-}$mice contained a higher frequency of IFN-producing cells $(P<0.001)$. One of 3 experiments is presented.

trap cassette encompassing an engrailed- 2 splice acceptor sequence, a $\beta$-gal cassette, and a neomycin gene resistance cassette. For negative selection, a viral thymidine kinase cassette was inserted into the targeting vector. Gene targeting in E14.1 ES cells was performed as described previously (35). Screening for homologous recombinant ES cell lines was performed by PCR using the primers HVEM-SP-5' (5'-TCCCTGAGGCTGAGAGGTTCC-3') and pJak2 (5'-CTGAAGAGGAGTTTACGTCCAG-3'). Two of 723 G418-resistant ES cell clones gave positive signals for homologous recombination and could be verified by Southern blot analysis (EcoRI digest) using the $5^{\prime}$ flanking probe. Single integration of the targeting vector was verified by Southern blot using a neomycin probe (NheI digest); here only 1 hybridizing band was detectable (data not shown). Both ES cell clones containing the correctly targeted HVEM allele were injected into C57BL/6 blastocysts. Germ-line transmission of the inactivated HVEM locus of both cell lines was confirmed by PCR and Southern blot analysis. Heterozygous mice were backcrossed 5 times into the C57BL/6 strain. At the N7 generation, homozygous offspring was obtained by intercrossing with $H V E M^{+/-}$mice. Genotyping for the HVEM alleles was performed by PCR using the primers HVEM-SP-5' and PJak2 for the inactivated allele and the primers HVEM-SP-5' and HVEM-SP-3'-WT (5'-AGAGGCAGCAGGGTCAGCTGG-3') for the WT allele. The mice were housed in a specific pathogen-free animal facility. For verification of the inactivation of the HVEM gene, a Northern blot analysis of spleen RNA was performed using the cDNA encompassing the extracellular domain of the murine HVEM.
Generation of anti-HVEM antibody. Rats were immunized 2 times with $100 \mu \mathrm{g}$ HVEM-mouse Ig fusion protein mixed with complete adjuvant and then boosted with $100 \mu \mathrm{g}$ of soluble HVEM-Ig 3 days before fusion. After fusion, 56 clones binding to HVEM-Ig were selected by ELISA, and, of those, 2 that specifically bind to HVEM but not mouse Ig were further characterized as IgM with $\kappa$ chain. They bind to HVEM-positive cells but not HVEM-negative cells.

Generation of recombinant LIGHT with modified extracellular domain. An extracellular fragment of murine LIGHT (85-240 amino acids) was amplified by PCR and inserted into Pcmv-flag-1 vector (Sigma-Aldrich) at HindIII/BamHI in frame with the flag tag (Pcmv-flag-LIGHT). Pcmv-flagLIGHT was used as template to amplify a flag-LIGHT fragment that was subsequently inserted into mammalian expression vector $\mathrm{pEf}$-Bos (provided by W.M. Yokoyama, Washington University, St. Louis, Missouri, USA). CHO cell line was cotransfected with pEf-Bos-flag-LIGHT and pcDNA3.1hygromycin by Lipofectamine reagent (Invitrogen Corp.). Transfectants were selected by $500 \mu \mathrm{g} / \mathrm{ml}$ hygromycin. Flag-LIGHT was purified from culture supernatant by anti-flag M2-agarose affinity gel.

ConA treatment. ConA (Sigma-Aldrich) was administered i.v. to the mice at different doses as indicated. Sera were collected at the indicated times for measurement of cytokines and liver enzymes. Plasma AST and ALT activities were determined using a Reflotron Plus according to the manufacturer's procedure (Roche Diagnostics Corp.).

Histopathology and TUNEL staining. Spleen and liver tissues were fixed in $10 \%$ formalin solution and then paraffin-embedded. H\&E staining on sections of embedded tissues was performed according to standard procedure. Cells undergoing apoptosis were detected using a modified TUNEL method at the core immunohistochemistry facility at the University of Chicago. Briefly, the spleen sections were incubated with $2 \mathrm{mM}$ digoxigeninconjugated dUTP (Chemicon Inc.) and $5 \mathrm{U}$ of terminal deoxynucleotidyl transferase (TdT) in TdT buffer $\left(0.5 \mathrm{M}\right.$ cacodylate [pH 6.8], $1 \mathrm{mM} \mathrm{CaCl}_{2}$, $0.5 \mathrm{mM}$ DTT, $0.05 \% \mathrm{BSA}, 0.15 \mathrm{M} \mathrm{NaCl}$ ). After washing in Tris-buffered saline, sections were incubated with HRP-conjugated anti-sheep Ig antibody (Jackson ImmunoResearch Laboratories Inc.). Color development for bound HRP was with 3-amino-9-ethylcarbazole (Sigma-Aldrich). Sections were then counterstained.

In vitro T cell proliferation. Splenocytes $\left(2 \times 10^{5}\right.$ per well) from C57BL $/ 6$ mice and $H V E M^{-/-}$mice were cultured in flat-bottomed 96-well plates with 0.3 , $0.6,1.2$, and $2.4 \mu \mathrm{g} / \mathrm{ml} \mathrm{ConA}$ or anti-CD3 for 48 hours. Supernatants were collected at 48 hours for cytokine measurement, and then cultured cells were pulsed with $0.5 \mu \mathrm{Ci}$ of ${ }^{3} \mathrm{H}$-thymidine for 16 hours before harvesting. In some experiments, $\mathrm{T}$ cells purified by MACS beads (Miltenyi Biotec) were cultured at $1 \times 10^{5}$ per well. For antigen stimulation, DLN cells were isolated 10 days after immunization and cultured in flat-bottomed 96-well plates at a concentration of $5 \times 10^{5}$ cells per well with various concentrations of MOG peptides. Plates were pulsed with ${ }^{3} \mathrm{H}$-thymidine (Amersham Pharmacia Biotech) at $0.5 \mu \mathrm{Ci} /$ well on day 2 (for anti-CD3) or day 4 (for peptide) of culture for the final 18 hours. Incorporation of thymidine into DNA was measured by liquid scintillation counting, and the mean was calculated from 3-4 wells.

Flow cytometry analysis. Single-cell suspensions of splenocytes and thymocytes were collected and stained with PE-anti-CD8, CyChrome-antiCD4, FITC-anti-CD69, CyChrome-anti-CD44, PE-anti-B220, and PE-anti$\mathrm{CD} 3$ antibodies (BD) and biotin-labeled anti-HVEM $\mathrm{mAb}$ in PBS plus $0.01 \%$ $\mathrm{NaN}_{3}$ for 30 minutes at $4{ }^{\circ} \mathrm{C}$. For biotin-labeled antibody, streptavidinCyChrome (BD) was used as secondary antibody. After incubation with antibodies, the cells were washed and analyzed on a FACScan (BD).

Cytokine measurement. Cytokine bead assay (BD Biosciences Pharmingen) or sandwich ELISA (BD Biosciences - Pharmingen) was used to test the cytokine level of the culture supernatants or sera according to the manufacturer's instructions. 
Induction of EAE. To induce EAE by active immunization, female $\mathrm{C} 57 \mathrm{BL} / 6$ mice and $H V E M^{-/-}$mice were immunized s.c. at 4 sites on the flank on days 0 and 7 with a total of $100 \mu$ g of MOG 35-55 peptide emulsified in an equal volume of CFA (GIBCO; Invitrogen Corp.) containing $1 \mathrm{mg} / \mathrm{ml}$ of Mycobacterium tuberculosis H37 RA (Difco Laboratories). MOG 35-55 peptide (MEVGWYRSPFSRVVHLYRNGK) was synthesized by Alpha Diagnostic International Inc. The peptides were more than $86 \%$ pure, as determined by HPLC. Mice also received i.v. injections of $200 \mathrm{ng}$ of pertussis toxin (List Biological Laboratories Inc.) on day 0 with the peptide. Mice were scored daily for signs of EAE according to the following clinical scoring system: 0 , no clinical signs; 0.5 , partial loss of tail tonicity; 1 , complete loss of tail tonicity; 2 , flaccid tail and abnormal gait; 3 , hind leg paralysis; 4 , hind leg paralysis with hind body paresis; 5 , hind leg and foreleg paralysis; 6 , death.

Detection of IFN- $\gamma$-producing cells by ELISPOT. Serial 5 -fold dilutions of DLN cells were plated in triplicate into 96-well plates (ELISPOT kit; BD) in the presence of $25 \mu \mathrm{g} / \mathrm{ml} \mathrm{MOG}$ and incubated overnight at $37^{\circ} \mathrm{C}$. Bound IFN- $\gamma$ was detected by incubation with alkaline phosphataseconjugated goat anti-mouse IFN- $\gamma(\mathrm{BD})$ at room temperature for 3 hours. Color development was performed with nitroblue tetrazolium substrate solution (BD).

\section{Acknowledgments}

This research was supported by grants from the NIH (R01 HD37104, R01 DK58897, and P01 CA09296-01) and the Deutsche Forschungsgemeinschaft (Pf 259/2-6 and Sonderforschungsbereiche 575,576 ). We thank Carl Ware for helpful discussion.

Received for publication August 9, 2004, and accepted in revised form December 14, 2004.

Address correspondence to: Klaus Pfeffer, Institute of Medical Microbiology, Heinrich-Heine-Universität Düsseldorf, Düsseldorf, Germany. Phone: 0211-81-12459; Fax: 0211-81-15906; E-mail: klaus.pfeffer@uni-duesseldorf.de. Or to: Yang-Xin Fu, Department of Pathology, University of Chicago, Chicago, Illinois 60637, USA. Phone: (773) 702-0929; Fax: (773) 834-8940; E-mail: yfu@midway. uchicago.edu.
1. Croft, M. 2003. Co-stimulatory members of the TNFR family: keys to effective T-cell immunity? Nat. Rev. Immunol. 3:609-620.

2. Chen, L. 2004. Co-inhibitory molecules of the B7CD28 family in the control of T-cell immunity. Nat. Rev. Immunol. 4:336-347.

3. Sharpe, A.H., and Freeman, G.J. 2002. The B7CD28 superfamily. Nat. Rev. Immunol. 2:116-126.

4. Salomon, B., and Bluestone, J.A. 2001. Complexities of CD28/B7: CTLA-4 costimulatory pathways in autoimmunity and transplantation. Annu. Rev. Immunol. 19:225-252.

5. Mauri, D.N., et al. 1998. LIGHT, a new member of the TNF superfamily, and lymphotoxin alpha are ligands for herpesvirus entry mediator. Immunity. 8:21-30.

6. Tamada, K., et al. 2000. Modulation of T-cell-mediated immunity in tumor and graft-versus-host disease models through the LIGHT co-stimulatory pathway. Nat. Med. 6:283-289.

7. Zhai, Y., et al. 1998. LIGHT, a novel ligand for lymphotoxin $\beta$ receptor and TR2/HVEM induces apoptosis and suppresses in vivo tumor formation via gene transfer. J. Clin. Invest. 102:1142-1151.

8. Rooney, I.A., et al. 2000. The lymphotoxin-beta receptor is necessary and sufficient for LIGHTmediated apoptosis of tumor cells. J. Biol. Chem. 275:14307-14315.

9. Shaikh, R.B., et al. 2001. Constitutive expression of LIGHT on T cells leads to lymphocyte activation, inflammation, and tissue destruction. J. Immunol. 167:6330-6337.

10. Wang, J., et al. 2001. The regulation of T cell homeostasis and autoimmunity by $\mathrm{T}$ cell-derived LIGHT. J. Clin. Invest. 108:1771-1780. doi:10.1172/ JCI200113827.

11. Wang, J., et al. 2004. Dysregulated LIGHT expression on $\mathrm{T}$ cells mediates intestinal inflammation and contributes to IgA nephropathy. J. Clin. Invest. 113:826-835. doi:10.1172/JCI200420096.

12. Yu, P., et al. 2004. Priming of naive T cells inside tumors leads to eradication of established tumors.
Nat. Immunol. 5:141-149.

13. Ye, Q., et al. 2002. Modulation of LIGHT-HVEM costimulation prolongs cardiac allograft survival. J. Exp. Med. 195:795-800.

14. Scheu, S., et al. 2002. Targeted disruption of LIGHT causes defects in costimulatory $\mathrm{T}$ cell activation and reveals cooperation with lymphotoxin beta in mesenteric lymph node genesis. J. Exp. Med. 195:1613-1624.

15. Tamada, K., et al. 2002. Cutting edge: selective impairment of CD8 $+\mathrm{T}$ cell function in mice lacking the TNF superfamily member LIGHT. J. Immunol. 168:4832-4835.

16. Marsters, S.A., et al. 1997. Herpesvirus entry mediator, a member of the tumor necrosis factor receptor (TNFR) family, interacts with members of the TNFR-associated factor family and activates the transcription factors NF-kappaB and AP-1. J. Biol. Chem. 272:14029-14032.

17. Granger, S.W., and Rickert, S. 2003. LIGHT-HVEM signaling and the regulation of $\mathrm{T}$ cell-mediated immunity. Cytokine Growth Factor Rev. 14:289-296.

18. Cauwels, A., et al. 2000. Protection against TNFinduced lethal shock by soluble guanylate cyclase inhibition requires functional inducible nitric oxide synthase. Immunity. 13:223-231.

19. Ferrara, J.L. 1995. Cytokine inhibitors and graft-versus-host disease. Ann. N. Y. Acad. Sci. 770:227-236.

20. Sun, R., Tian, Z., Kulkarni, S., and Gao, B. 2004. IL-6 prevents $T$ cell-mediated hepatitis via inhibition of NKT cells in CD4+ T cell- and STAT3-dependent manners. J. Immunol. 172:5648-5655.

21. Tamada, K., et al. 2000. LIGHT, a TNF-like molecule, costimulates $\mathrm{T}$ cell proliferation and is required for dendritic cell-mediated allogeneic $\mathrm{T}$ cell response. J. Immunol. 164:4105-4110.

22. Lenschow, D.J., Walunas, T.L., and Bluestone, J.A. 1996. CD28/B7 system of T cell costimulation. Annu. Rev. Immunol. 14:233-258.

23. Chambers, C.A., Kuhns, M.S., Egen, J.G., and Allison, J.P. 2001. CTLA-4-mediated inhibition in regulation of $\mathrm{T}$ cell responses: mechanisms and manipulation in tumor immunotherapy. Annu. Rev. Immunol. 19:565-594.

24. Dong, H., Zhu, G., Tamada, K., and Chen, L. 1999. B7-H1, a third member of the B7 family, co-stimulates T-cell proliferation and interleukin-10 secretion. Nat. Med. 5:1365-1369.

25. Tamura, H., et al. 2001. B7-H1 costimulation preferentially enhances CD28-independent T-helper cell function. Blood. 97:1809-1816.

26. Freeman, G.J., et al. 2000. Engagement of the PD-1 immunoinhibitory receptor by a novel B7 family member leads to negative regulation of lymphocyte activation. J. Exp. Med. 192:1027-1034.

27. Dong, H., et al. 2002. Tumor-associated B7-H1 promotes T-cell apoptosis: a potential mechanism of immune evasion. Nat. Med. 8:793-800.

28. Subudhi, S.K., et al. 2004. Local expression of B7-H1 promotes organ-specific autoimmunity and transplant rejection. J. Clin. Invest. 113:694-700. doi:10.1172/JCI200419210.

29. Chapoval, A.I., et al. 2001. B7-H3: a costimulatory molecule for $\mathrm{T}$ cell activation and IFN-gamma production. Nat. Immunol. 2:269-274.

30. Suh, W.K., et al. 2003. The B7 family member B7-H3 preferentially down-regulates $T$ helper type 1-mediated immune responses. Nat. Immunol. 4:899-906.

31. Chen, L. 2002. Antibody gene therapy: old wine in a new bottle. Nat. Med. 8:333-334.

32. Foell, J., et al. 2003. CD137 costimulatory T cell receptor engagement reverses acute disease in lupus-prone $\mathrm{NZB} \times \mathrm{NZW} \mathrm{F}_{1}$ mice. J. Clin. Invest. 111:1505-1518. doi:10.1172/JCI200317662.

33. Sun, Y., et al. 2002. Costimulatory molecule-targeted antibody therapy of a spontaneous autoimmune disease. Nat Med. 8:1405-1413.

34. Sedy, J.R., et al. 2005. B and T lymphocyte attenuator regulates $\mathrm{T}$ cell activation through interaction with herpesvirus entry mediator. Nat. Immunol. 6:90-98.

35. Neubauer, H., et al. 1998. Jak2 deficiency defines an essential developmental checkpoint in definitive hematopoiesis. Cell. 93:397-409. 